\title{
Helicobacter pylori: A Nobel pursuit?
}

\author{
Barry Marshall $M D^{1}$, Paul C Adams $M^{2}$
}

$\mathrm{I}_{\mathrm{R}}^{\mathrm{n}}$ n 2005, Barry Marshall and the Nobel prize in Physiology for their pioneering work on Helicobacter pylori. In the words of the Nobel Committee, they were honored "for their discovery of the bacterium Helicobacter pylori and its role in gastritis and peptic ulcer disease." The Committee added, "Thanks to the pioneering discovery by Marshall and Warren, peptic ulcer disease is no longer a chronic, frequently disabling condition, but a disease that can be cured by a short regimen of antibiotics and acid secretion inhibitors."

Today, Barry Marshall is the National Health Medical Research Council Senior Principal Research Fellow at the University of Western Australia. In recent years, his research has illuminated the patterns of Helicobacter infection in different populations around the world. It is probably the most widespread chronic infection in the world, and is nearly universal in the world's poorest countries. Dr Marshall hopes to see the insidious corkscrew organism controlled to the point where it can no longer pose a threat to the life and health of men, women and children anywhere in the world. Dr Marshall continues to practice as a gastroenterologist in Perth, Australia.

PA: Can you remind us of the details of the famous story of you ingesting $\mathrm{H}$ pylori? Did it occur to you that there is a legacy of self-experimentation in medical history such as Marie Curie?

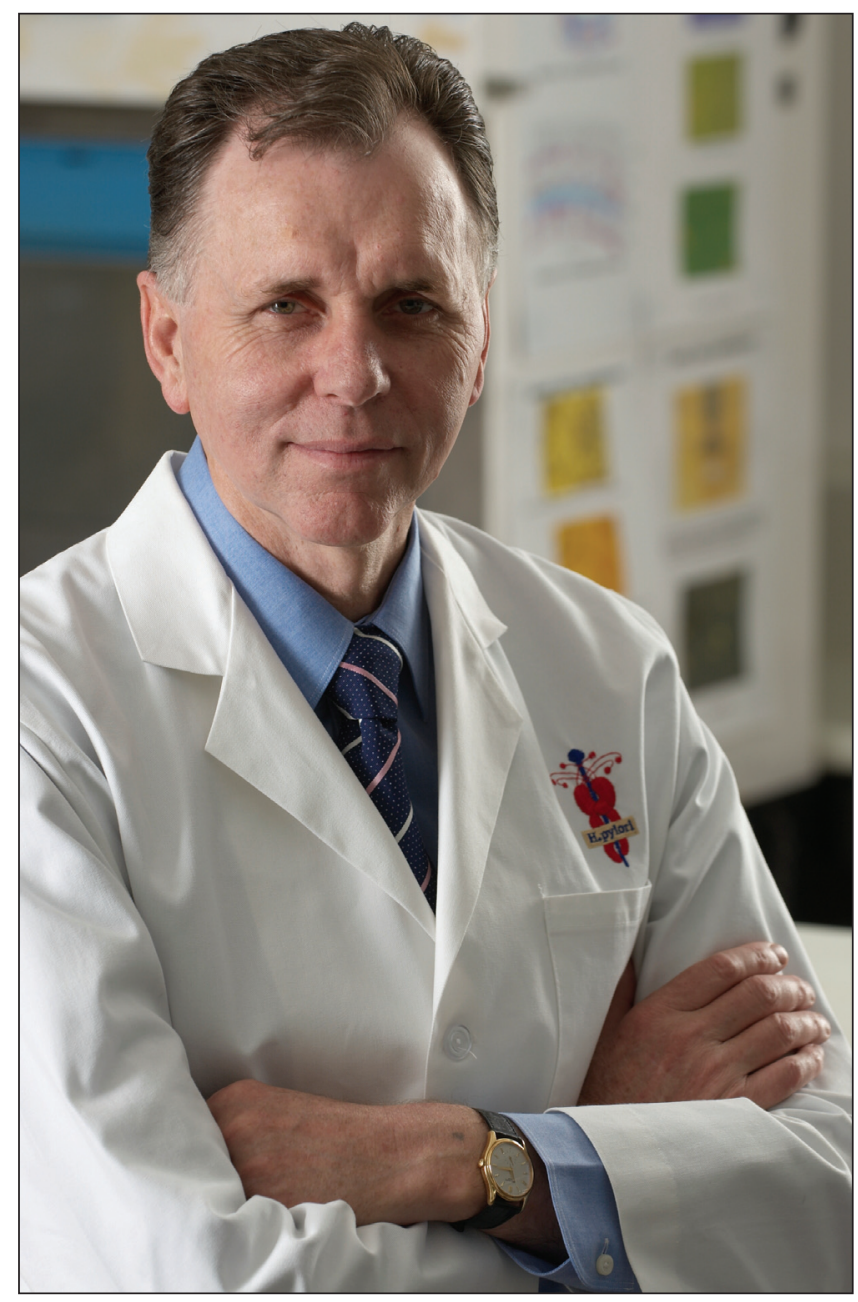

Dr Barry Marshall is the National Health Medical Research Council Senior Principal Research Fellow at the University of Western Australia, Australia
BM: I was aware of famous selfexperiments because I read the history of John Hunter's selfinfection with gonorrhea and syphilis (which may have caused his death years later). However, I had been arguing with the skeptics for two years and had no animal model that could prove $H$ pylori was a pathogen. If I was right, then anyone was susceptible to the bug and would develop gastritis and maybe an ulcer years later. So I expected to develop an asymptomatic infection. The experiment was planned with a culture from a patient with dyspepsia and confirmation that it was sensitive to metronidazole. Then I underwent endoscopy in early July 1984 to confirm that I was negative for $H$ pylori. Three weeks later, I drank the 'brew' which was a suspension of two culture plates of the organism. If only I knew that people would be so interested, I would have taken a photograph! After five days, I started to have bloating and fullness after the evening meal, and my appetite decreased. My breath was bad and I vomited clear watery liquid, without acid, each morning at approximately 06:00. Then, a follow-up endoscopy showed severe active gastritis with polymorphonuclear infiltrate and epithelial damage. Evidently, $\mathrm{H}$ pylori was a pathogen for normal people. The ulcer did not merely set you up for catching the infection. People with asymptomatic $H$ pylori were 'carriers' and most people did not have ulcers from the bacterium. Gastritis was explained.

\footnotetext{
${ }^{1}$ University of Western Australia, Subiaco, Australia; ${ }^{2}$ University Hospital, London, Ontario

Correspondence: Dr Barry Marshall, Nobel Laureate, University of Western Australia, PO Box 2001, Subiaco, Western Australia 6904.

fax618-9382-3713, e-mail bmarshall@hpylori.com.au
}

Received and accepted for publication July 31, 2008. 
After 14 days, I repeated the endoscopy and then, before the results were known, began taking antibiotics (on my wife's orders!). However $H$ pylori were not seen on that biopsy so I might have already had a spontaneous cure. Robin Warren believes that the bacteria were still lurking and would have been detected on culture, but by then I was already treated. The paper was published in the third person, but it gradually became known that the 'male volunteer' was me.

PA: There have now been many patients treated for $\mathrm{H}$ pylori, are we starting to see changes in the natural history of peptic ulcer disease or gastric cancer?

BM: Nowadays idiopathic peptic ulcer is rare in Australia, usually confined to immigrants who carry $H$ pylori from their home country. Ulcers that result from nonsteroidal anti-inflammatory drug use now make up more than $50 \%$ of the cases, which means that the total ulcer population has been diminished by $70 \%$. In Australia, that represents a savings of $\$ 300$ million per year. Similarly, gastric cancer is usually in immigrant populations. But gastric cancer is not as clearly preventable as peptic ulcers. With a healthy diet, acid levels are maintained throughout life and may protect from cancer. So recent studies in China and Japan have shown less cancer risk after H pylori cure, but not complete absence of risk. Most of the studies have been underpowered probably because, even in China nowadays, diet is better and gastric carcinogens are less common. Therefore, the control groups have a lower cancer incidence than one would expect from historical data.

PA: If $H$ pylori is ingested, why are patients not constantly re-infected?
BM: In developed countries, there is no environmental source of $\mathrm{H}$ pylori, and infection from family members is usually during the first years of life. Although infection from partners occurs in adults, the rate is probably less than $2 \%$ per year.

PA: There are many more patients with $H$ pylori than there are with ulcers or gastric cancer.

BM: The ulcer lifetime risk may be $20 \%$ but the cancer lifetime risk is less, probably $1 \%$ to $5 \%$. Cancer risk is affected by socioeconomic factors, nutrition and perhaps diet. For whatever reason, cancer risk is less in developed countries.

PA: I know you have been considering the impact of $\mathrm{H}$ pylori on other medical conditions. Where do you see this work heading in the future?

BM: I think $H$ pylori will be proven to be a slight risk for iron deficiency anemia, cardiovascular disease and various immune-mediated disorders. Presently, most of the associated diseases are 'unproven' when studied carefully in a prospective trial. However, if a disease does not have a known etiology, or a cure available, then treatment of $H$ pylori is a rather simple thing to try. Far easier than chemotherapy which might be the alternative for a disorder such as idiopathic thrombocytopenic purpura. What is the future for $\mathrm{H}$ pylori? Ultimately we will have a complete understanding of its transmission and will be able to control it, even in developing countries. Dietary and 'probiotic' prevention might work, although at present, these are treatment concepts rather than definitive therapies. In Japan, it seems likely that a total eradication program could be implemented to wipe out $\mathrm{H}$ pylori completely. 


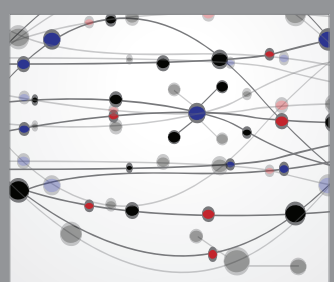

The Scientific World Journal
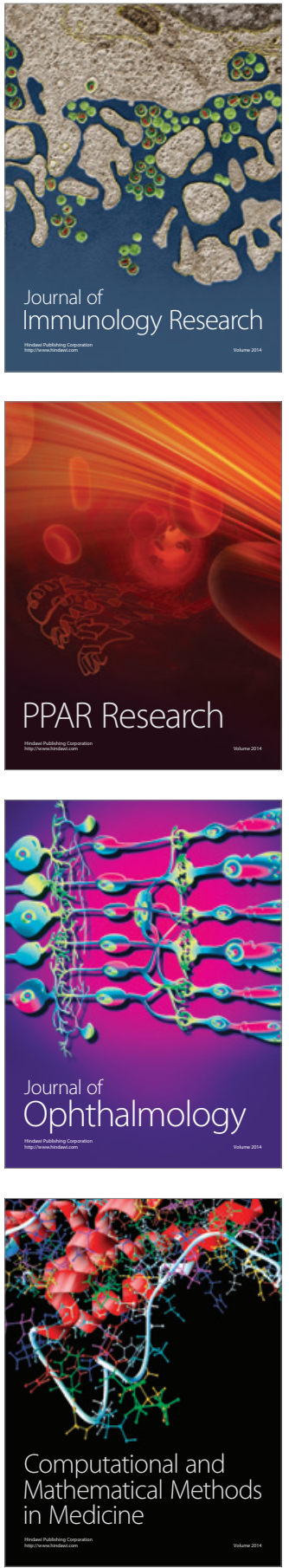

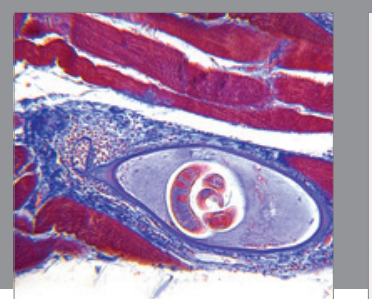

Gastroenterology Research and Practice

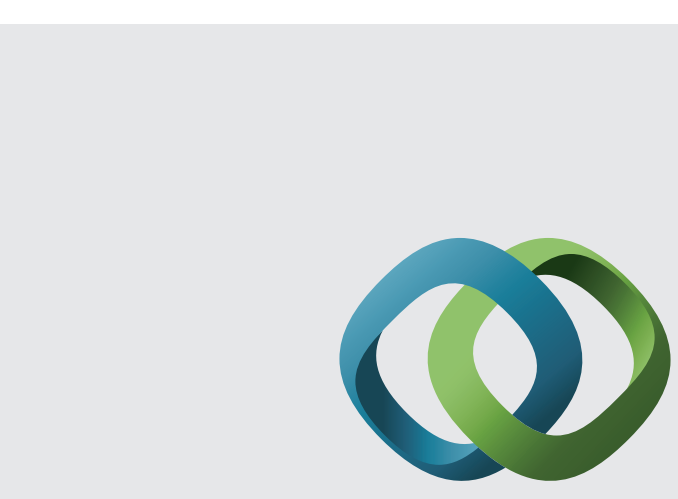

\section{Hindawi}

Submit your manuscripts at

http://www.hindawi.com
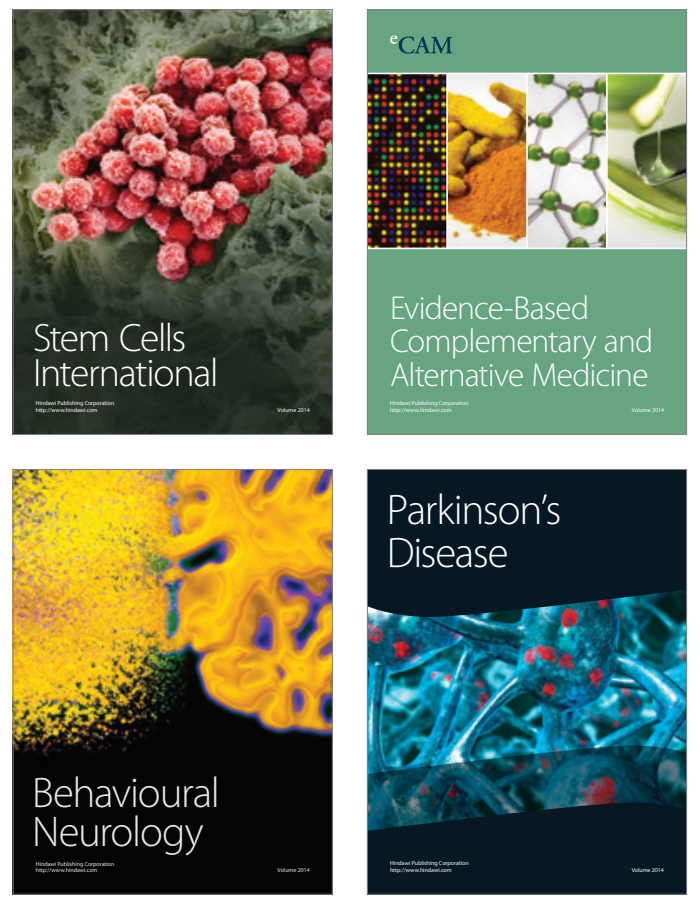
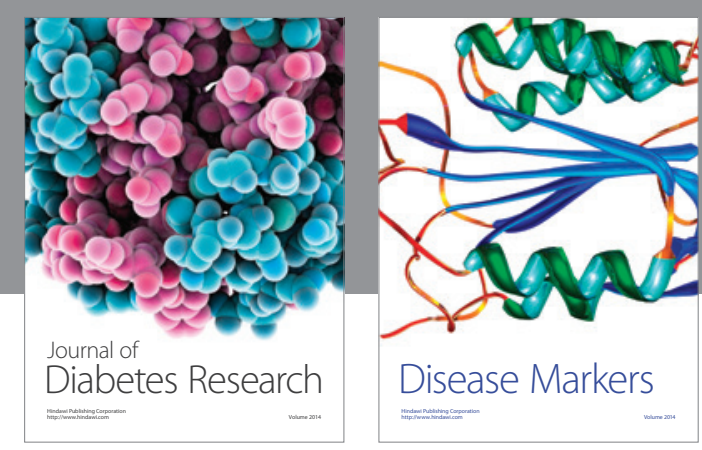

Disease Markers
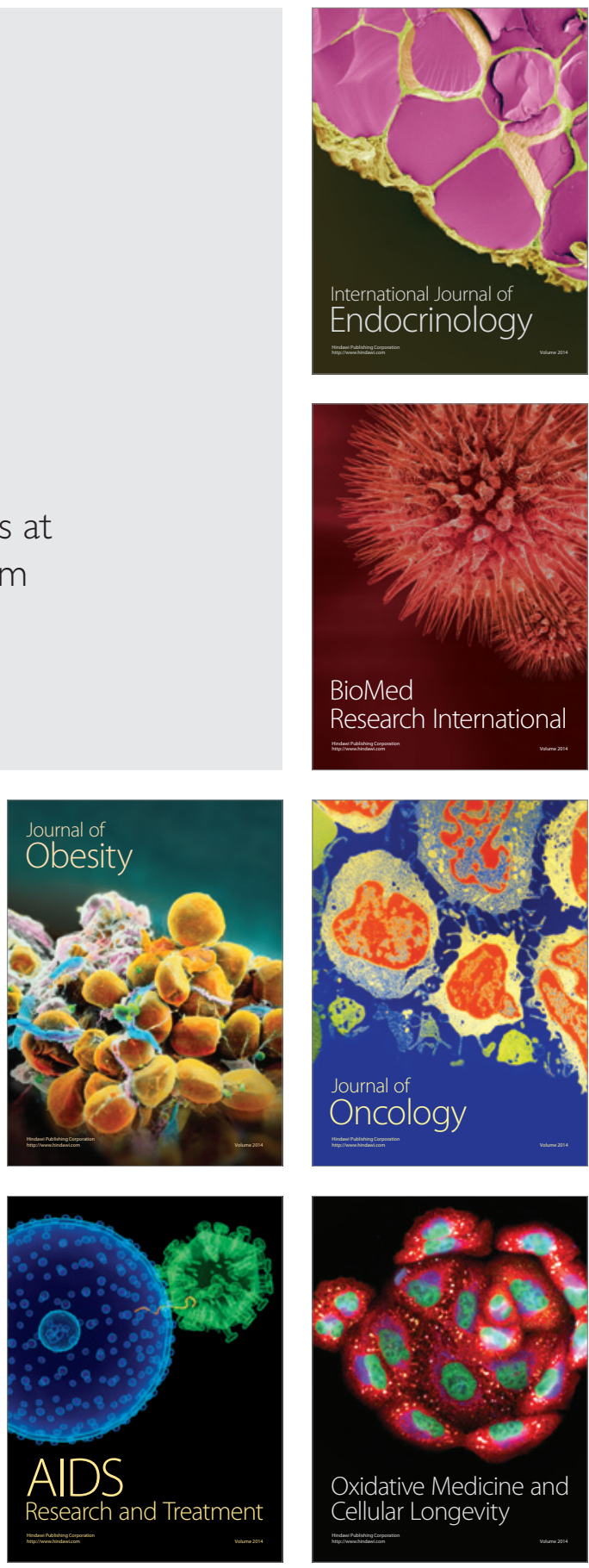